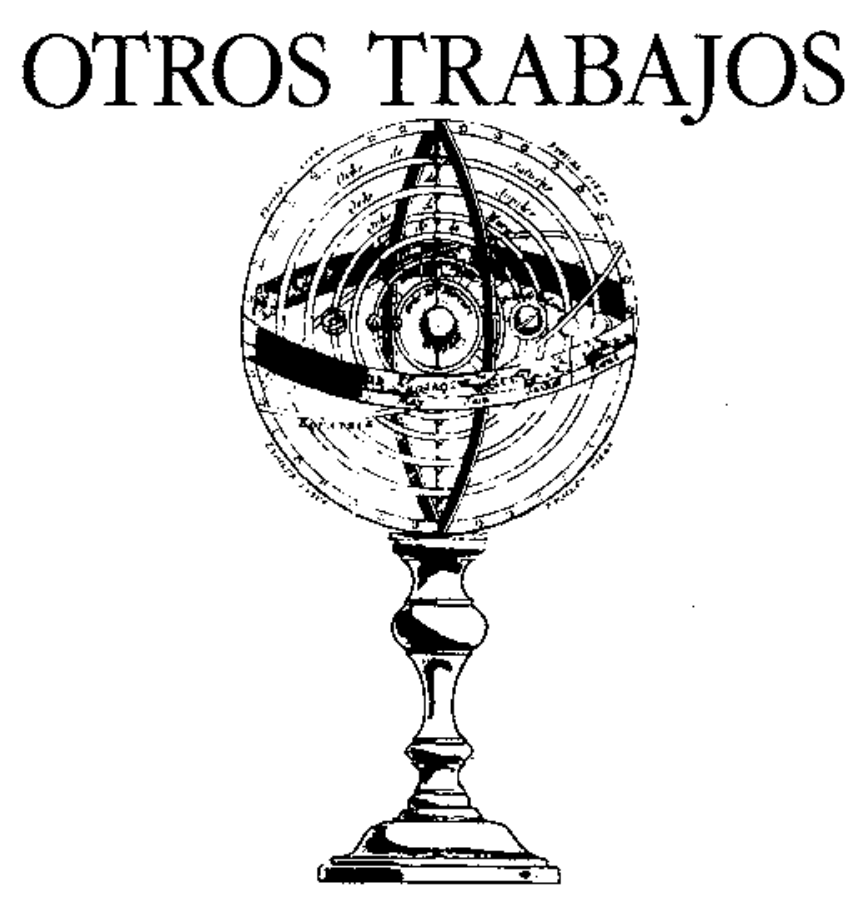

\title{
ENSEÑANZA Y APRENDIZAJE DE LA MODELIZACION
}

MARTINAND, J.L.

Lirespt, Université Paris 7

Versió castellana de Francisco Carbonell Gisbert. I.B. «Fco. Figueras Pacheco», Alicante

\section{SUMMARY}

A general outline of a research program on the teaching/learning of modelization, being carried out at the moment, is presented. Particular reference is made to the difficulties of learning modelization considering the different types of activities, rhythm, etc. which are suitable to help such learning.

As an instance, both relationships force-energy and the atomic model were chosen.

\section{PRESENTACION GENERAL}

\subsection{Importancia del uso de modelos}

Para el dominio de la realidad natural, técnica, económica o social contemporánea, el uso de los modelos reviste una importancia fundamental. Son en efecto medios de una aprehensión de dos aspectos mayores de esta realidad:

- más allá de los objetos y propiedades familiares, de los procesos fácilmente accesibles y modificables, es preciso imaginar to oculto, y reemplazar las representaciones primeras por variables, parámetros, rela- 
ciones entre variables. Las nuevas representaciones adquieren entonces un carácter relacional e hipotético.

- más allá de las situaciones simples, es preciso de senredar madejas complejas y ast mismo identificar y manipular buenos sistemas describiéndolos con variables de estado y de interacción, relaciones internas entre estas variables, valores de tensiones exteriores. Las nuevas representaciones adquieren entonces un carácter sistemático.

Este doble carácter sistemático e hipotético es constitutivo de los modelos que construyen las ciencias y que los alumnos deben pues apropiarse, (L. Von Bertalanffy, J.l. Lemoigne, H. Simon, B. Walliser.)

\subsection{Dificultades del aprendizaje de la modelización}

Las ciencias físicas son evidentemente y desde hace tiempo grandes constructoras y manipuladoras de modelos; los pasos, las actitudes de modelización juegan un papel mayor, apoyándose a la vez en la teoría y la experimentación. Podríamos pues esperar a ver conceder a la modelización una gran importancia en las actividades escolares en el colegio y en el instituto. Es sin duda el caso sobre el plan de las intenciones proclamadas pero en la realidad de las clases y de los manuales, es la imposición de un punto de vista y de un modo de descripción que domina: punto material, vector fuerza, rayos luminosos, átomo, etc..., no son presentados como modelos, es decir representaciones constuidas, "calculables», más o menos «simplificadas», ni incluso representaciones, sino como la realidad directamente visible.

En estas condiciones, es fácil comprender los fracasos en los niños a quienes les cuesta mucho trabajo unir los modelos presentados a las situaciones reales del laboratorio o de la vida fuera de la escuela (de aquí un cierto rechazo), y que no llegan a hacer funcionar éstos como construcciones hipotéticas y heurísticas, sino como dogmas definitivos y cerrados.

\subsection{Hacia una enseñanza de la modelización}

No se trata de concluir de estas dificultades la supresión de toda enseñanza física antes del Instituto. Pero es importante reflexionar más profundamente en la modelización para transformar los contenidos de la ensehlanza: ¿Qué se hace modelizando? ¿Qué es lo que esto aporta? ¿Cómo funciona esto en el pensamiento científico?. Es preciso también conocer mejor cómo los niños se las arreglan cuando modelizan espontáneamente o buscan apropiarse un modelo (problemas de «lectura" de la realidad según un punto de vista, de descripción de ésta, de construcción de representaciones, de manipulación de simbolismos, de confrontación con la realidad).

Se trata por último de preguntarse qué tipos de actividades especificas es posible proponer en clase; icon qué medios? ¿Qué sugèstiones de progresiones, de rit- mos de aprendizaje, pueden ser propuestos a los maestros para organizar la enseñanza?

Un tal programa de trabajo supone conjugar un análisis epistemológico del problema, la preparación, la conducción y evaluación de ensayos didácticos en clase, la observación precisa de marchas individuales o en pequentos grupos.

\subsection{Elección de los campos: fuerza-energía, modelo atómico}

A todas las disciplinas cientfficas y tecnológicas les con. cierne a priori la modelización y la tarea de mejorar la enseñanza y el aprendizaje se impone a todas.

Entre ellas las ciencias físicas, y particularmente la mecánica (cuerpos rígidos y medios continuos), han sido sin duda las primeras en la historia en poner en evidencia, de forma explícita, la modelización y a dominarla. Aunque encontremos ahora los mismos problemas tanto en termodinámica, química, biológica y ciencias sociales, parece pues juicioso dirigir el esfuerzo de la investigación didáctica a propósito de la modelización sobre las ciencias físicas.

Tanto por las analogias y convergencias, como por las diferencias de cuestiones que plantean a este respecto (magnitudes y relaciones entre magnitudes, papel del espacio real y representativo, modos de simbolizacion) dos campos parecen particularmente interesantes para este estudio:

- la mecánica-energética, con la esquematización de fuerzas y el concepto de energia,

- la estructura de la materia y la reacción química con los modelos atómicos.

Con la introducción de las ciencias físicas en la ensenanza secundaria elemental (11-14 años) y en la escuela primaria, las necesidades y posibilidades de conceptualización y de modelización aparecen muy pronto (Martinand, 1984; Mehuet, 1982). Pero teniendo en cuenta los programas actuales y las investigaciones anteriores, es razonable interesarse por las clases que van desde $4^{\circ}$ (13-14 años) al final de la secundaria. Más precisamente, se trata de estudiar según la orientación presentada arriba, los períodos de primera aproximación sistemática:

- la fuerza y la energía, desde $3^{\circ}$ (14-15 años) al final (18 años).

- el modelo atómico, en $4^{\circ}, 3^{\circ}$ y $2^{\circ}$.

\subsection{Objetivo de la investigación: conocimiento de los alumnos y proposiciones curriculares}

En cada uno de estos campos, y teniendo en cuenta el hecho que la confrontación entre los dos campos debería permitir enriquecer y generalizar los resultados obtenidos, se trata para nosotros: 
- de hacer el balance del problema de la modeliza. ción en la ensetanza (estudio de programas y manuales),

- de preparar, conducir y observar secuencias de enseñanza-aprendizaje con algunos alumnos,

- de preparar y evaluar, con enseñantes, actividades de clase.

El objetivo de este trabajo es la puesta a punto de ayudas didácticas o de materiales para la formación de maestros (en particular: descripción y análisis de actividades sobre la modelización), propuestas «curriculares») (sugerencias de modificaciones de contenidos de programas, teniendo en cuenta la perspectiva a largo plazo que requiere el aprendizaje de la modelización).

Presentaremos ahora la situación del tema en cada uno de los dos campos elegidos y el plan de investigación.

\section{LA MODELIZACION EN LA ESTRUCTU. RA DE LA MATERIA}

\subsection{Situación actual del tema propuesto}

La enseñanza de nociones relativas a la estructura de la materia y su papel en la modelización de fenomenos físico-químicos es, después de los años 60 , el objeto de programas de enseñanza, de innovaciones y de inves. tigaciones en Francia y en el extranjero.

Un primer análisis de enseñanzas realizadas en este campo pone en evidencia su diversidad en los siguientes aspectos:

- la complejidad del modelo propuesto

- papel que le es atribuido. En ciertos casos es utilizado para explicar y prever los resultados experimentales, en otros, funciona más bien como una imagen, es poco utilizado de forma operatoria.

- estrategias didácticas propuestas: elaboración por los alumnos mismos de un modelo (proyecto danés "Ask Nature» por ejemplo) o al contrario transmisión por enseñanza (proyecto escocés) «Science for the 70'Sy por ejemplo).

Estudios llevados a cabo por Piaget y sus colaboradores (Piaget-Bliss) (Piaget-Inhelder 1962) describen diferentes estados de elaboración de un modelo particular de la materia por ninos de 10 a 13 años. Este modelo «espontáneo» posee un carácter operatorio limitado (disoluciones).

Ciertas investigaciones Good et al 1979; Godstein, 1978) establecen una relación entre el acceso al estado formal en el sentido piagetiano del término y la utilización fructífera de un modelo particular en química.

Ciertos trabajos (Bensaude y otros, 1984) muestran la importancia de la elección del vocabulario durante tal enseñanza, ciertas palabras son utilizadas por los alum. nos con preferencia a otras.

De manera general, los estudios lievados a cabo en este campo permiten delimitar los obstáculos encontrados durante la enseñanza de tales modelos. Una de las dificultades así encontrada puede ser formulada en términos de rechazo de la existencia del vacio (Mehuet, 1982) o de representación continua de la materia (Pfunt, 1981). Otra dificultad puede ser formulada en términos de rechazo de la noción de interacción entre partículas (Mehuet, 1982) o de transmision a las particulas de las propiedades macroscópicas (Driver, 1984, Sere, 1984).

\subsection{Plan de investigación}

\subsubsection{Objetivo general de los trabajos}

En los programas franceses actuales, la representación de tales modelos está claramente excluida en las clases de $6^{\circ}$ y $5^{\circ}$. Las primeras nociones sobre la estructura de la materia son introducidas a propósito de los metales y de las soluciones iónicas. Esto conduce pues a presentar el átomo como constituido de un núcleo y de electrones. La discontinuidad de la materia no está considerada como objetivo de un aprendizaje especifico. Es más bien presentada en los programas y obras escolares, como una evidencia. Su aceptación por los alumnos no es casi puesta en duda.

Uno de los objetivos de nuestro trabajo consiste pues en observar la adquisición de esta noción de discontinuidad por los alumnos de los colegios e institutos franceses, en el cuadro de los programas actuales. Esto nos permitirá precisar la amplitud de las necesidades para un aprendizaje específico de esta noción y proponer elementos para la construcción de tal aprendizaje, si éste se comprobara necesario.

\subsubsection{Programa de los trabajos}

Primer anto (1985):

Estudio de los razonamientos de los alumnos de colegio e instituto a propuesta de la estructura de la materia. Las nociones abordadas estarán limitadas a la divisibilidad de la materia por los métodos ff́sicos simples y a la existencia del vacio.

Primer y segundo trimestre 1985:

- Elección de las situaciones experimentales soporte de cuestionarios

- Elaboración de cuestionarios

- Recogida de datos: entrevistas individualescuestionarios escritos.

Tercer y cuarto trimestre 1985 - Primer trimestre 1986

- Análisis de los datos: dos direcciones principales del análisis.

- ¿Hay una cierta homogeneidad de respuestas para un 
niño dado y asf pues niños más bien "continuistas" y nifos más bien «discontinuistas» con relación a las situaciones experimentales propuestas?

- ¿Entre las situaciones experimentales propuestas, algunas conducen más que otras a interpretaciones en términos de discontinuidad?

- Elabóración de secuencias de enseñanza

Esta elaboración se basará en los resultados obtenidos precedentemente, particularmente a lo que concierne a la elección de situaciones experimentales de referencia y la elección del método. Este podrá comportar fases de inducción del modelo por los alumnos, fases de presentación de un modelo a partir de representaciones convenientemente elegidas y fases de generalizacion, ensanchamiento del campo de referencia experimental. La aplicación en las clases estará precedida de ensayos "en laboratorios" con pequertos grupos de alumnos.

Segundo trimestre 1986:

- Aplicación y observación de estas secuencias de enseftanza. Se observará las actividades experimentales, las producciones verbales y escritas de los alumnos en las diferentes fases indicadas anteriormente.

Tercer y cuarto trimestre:

- Análisis de datos recogidos en el curso de las secuencias de ensefianza.

\section{FUERZA-ENERGIA}

\subsection{Situación del tema}

La investigación que proponemos empezar se apoya sobre dos tipos de trabajos anteriores del laboratorio.

1- Una práctica de la formación de los enseñantes en el transcurso de encuentros de reciclaje efectuados en la Universidad de París y en la Academia de Creteil. Estos encuentros (stages) han sido ocasión, por una parte para hacer un análisis en profundidad del campo conceptual concernido, teniendo en cuenta los límites impuestos por el programa desde $3^{\circ}$ al curso terminal y por otra parte, para poner a punto situaciones experimentales susceptibles de preguntas para los ensefiantes y los alumnos, y para las cuales es posible proponer diferentes tipos de análisis con objeto de compararlos (análisis evidentes de tipo causal, análisis en términos de interacciones, análisis en términos de fuerza y energía) y de trabajar de manera pertinente sobre la noción de sistema.

2- Por otra parte, una reciente tesis de tercer ciclo (Khraibani, 1984) propone dar cuenta de las conductas de los alumnos ante la enseffanza en situaciones experimentales del mismo tipo que aquellas sobre las cuales hacemos trabajar a los ense-
Ilantes en los encuentros de perfeccionamiento; esto nos permite precisar cuáles son los saberes y los "saber-hacer» que permiten iniciar una enseñanza de la mecánica. Las situaciones estudiadas son simples, en el sentido que ponen en juego un pe queño número de objetos de la vida corriente, pero utilizados a la vez de forma inhabitual (báscula pesa personas, bast $n$, pared, etc...).

Otros estudios llevados a cabo en este terreno:

a) fuerza

Existen numerosas investigaciones llevadas a cabo con estudiantes que han obtenido el bachillerato. Se centraron sobre los obstáculos encontrados por estos estudiantes en la comprension de distintas situaciones experimentales que ponen en juego la relación entre fuerza y movimiento.

El estudio más importante llevado a cabo en Francia sobre este tema es el de Viennot (1979). El razonamiento espontáneo en dinámica elemental. Existe una revisión crítica de las investigaciones europeas y anglosajonas sobre este tema, hecha por Mc Dermott, en las actas del Taller Internacional de la Investigación en Didáctica de la Física (McDermott 1984).

Por el contrario no existen investigaciones sobre una primera aproximación del concepto de fuerza por los alumnos de 15-16 anos. Y sobre todo todas estas investigaciones están concebidas en una perspectiva restringida a la dinámica.

b) energía

Contrariamente a la situación de las investigaciones sobre el tema de la mecánica, existe poco trabajo de investigación didáctica a propósito del concepto de energía que pueda conducir a modificaciones de la enseñanza.

Ciertos autores se interesan por las representaciones que se hacen los alumnos de la energfa: J. Agraba (1982-83) en los alumnos de los cursos medios de la escuela elemental, D.M. Watts y J. Solomon en los alumnos de colegio y de Instituto, R. Duit (1981) en los estudiantes al principio de los estudios universitarios.

Otros autores presentan algunas secuencias de enseftanza, más o menos amplias, sobre el tema energía, a partir de actividades experimentales: Agabra (1982-83) y Genling y Temme (1982), para alumnos de cursos medios, Hulin (1982) para alumnos de instituto.

\subsection{Plan de investigación}

\subsubsection{Objetivo general}

Es en $3^{\circ}$, para alumnos de 15-16 aftos, cuando se introducen a la vez los conceptos de fuerza y energía, conceptos fundamentales sobre los que se basan muchas ensenanzas posteriores.

Son dos conceptos diffciles de aprender para los que 
se plantea de forma aguda el problema de su representación al mismo tiempo que el de la representación de los objetos sobre los cuales actúan. El paso de la percepción inmediata de un objeto a la representación de éste no es jamás tarea fácil; depende en efecto del dominio de la exploracion considerado (no se representa un automóvil de la misma manera según el concepto de fuerza o energía). Lo que es más, al mismo tiempo que una nueva representación de los objetos, es necesario elaborar una representación de lo que sucede en tre ellos.

Es la primera vez, en la enseñanza de la física, que los alumnos abordan una magnitud utilizada para describir las interacciones entre sistemas. A este propósito, surgen tres dificultades principales:

- Elaborar una nueva representación de los objetos que no borre la representación de los objetos surgida de una simple percepción sensorial o de su utilización familiar pero que, aunque se apoye en esta última, difiere fundamentalmente.

$\longrightarrow$ Reunir de forma arbitraria un conjunto de objetos y concebirlo como un sistema caracterizado por el conjunto de variables necesarias para describirlo completamente en el dominio considerado. Autorizarse así a operar una petición de la naturaleza de una forma que difiere de la percepción habitual no tiene raada de actividad banal y espontánea.

- Concebir una magnitud que describe algo que no existe más que entre sistemas (la fuerza) o una magnitud que no existe más que en los sistemas, pero que puede transferirse de un sistema a otro (la ener. gía). Abordar tales magnitudes no corresponde a un modo de pensamiento familiar a los alumnos de 15-16 aftos puesto que desde su más tierna edad, han sido entrenados a considerar las cualidades y los usos de los objetos que los rodean más bien que a juzgar las relaciones que hay entre ellos.

\subsubsection{Programa de los trabajos}

Nuestro proyecto consiste en proponer una enseñanza en $3^{\circ}$ que tenga en cuenta lo que, en las diferentes operaciones específicas referidas a los conceptos de fuer. za y energía, puede ser presentado con provecho a los alumnos.

Nuestras proposiciones estarán acompañadas de suge. rencias de secuencias didácticas para las que procederemos a una evaluación sobre la "factibilidad" en cla se y sobre las experiencias de los alumnos.

\section{- Primer año:}

Puesta a punto de secuencias didácticas y evaluación de estas secuencias desde el punto de vista de las progresiones que permiten para la ensentanza. Al lado del análisis de algunas pruebas pasadas colectivamente en clase, se procede a algunos estudios de casos individua- les. Se senala notablemene los alumnos que hayan adquirido un cierto dominio del concepto de fuerza o de energía. Se trata de comprender lo que haya podido facilitar este dominio, en comparación con los alumnos que no hayan sacado provecho de la misma manera de enseñar. Se facilita al máximo los intercambios entre los alumnos que son en algunos casos registrados y analizados. Procedemos nosotros mismos a entrevistas con estos dos tipos de alumnos.

\section{- Segundo año:}

A partir de los análisis efectuados en el curso del primer año, se podrá hacer propuestas de enseñanza que sean experimentadas y evaluadas en una clase (o más si las colaboraciones y los medios lo permiten). Para la evaluación serán tomadas en cuenta de una parte las condiciones de factibilidad de tal enseñanza, de otra parte una estimación de las adquisiciones de los alumnos en las condiciones ideales donde intentaremos situarnos. Se apuntarán las adquisiciones internas en el dominio de los conocimientos indicados y las transferencias posibles en otros dominios.

\section{COORDINACIÓN DE LOS TRABAJOS}

Los programas de los trabajos en los dos dominios, si hacen aparecer el paralelismo de las dos investigaciones, no hacen destacar las posibilidades ofrecidas para fecundaciones mutuas. Insistiremos ahora sobre dos momentos:

- Reflexión epistemológica general del laboratorio sobre las nociones de sistema y de modelo, en relacio. nes con otras investigaciones del laboratorio sobre la simulación con microordenadores, y el papel de la simbolización en la resolución de problemas, así como con los trabajos del Grupo de Investigaciones Coordinadas CNRS (matemáticos, físicos, psicólogos) «Didáctica y adquisición de conocimientos científicos».

- Análisis de las actividades didácticas de modelización propuestas en los programas y manuales.

- Confrontación (convergencias y divergencias) entre la modelización necesaria en la construcción de las magnitudes fuerza y energía y la modelización atómica de la estructura de la materia.

El conjunto de esta investigación se inscribe pues en el marco de las investigaciones curriculares apoyadas a la vez sobre las reflexiones fundamentales (epistemología y pedagogia del modelo), observaciones detalladas de procesos de enseflanza, aprendizaje, ensayos (concepción y evaluación) de propuestas curriculares. Ello debe pues desembocar en un mejor conocimiento de las dificultades y posibilidades de los alumnos a propósito de la modelización de los conceptos fuerza y energia y de la estructura atómica, ast como sobre ma. teriales $\mathrm{e}$ indicaciones didácticas (propuestas de programas y de actividades en clase, materiales y documentos, y medios de evaluación). 


\section{REFERENCIAS BIBLIOGRAFICAS}

AGABRA, J., 1982-83, Aster, No 19-20, INRP.

BENSAUDE, B. et autres 1984, Résultats d'une enquête sur quelques vocables d'orientation scientifique, Signes et discours dans l'education scientifique, sixièmes journées internationales sur l'éducation scientifique, A. Giordan et J.L. Martinand Editeurs, 441-448.

DRIVER, R., 1984, An approach to documenting the understanding of 15 years old british children about the particulate theory of matter, Recherches en Didactique de la physique: Les Actes du Premier Atelier International, Editions du CNRS, 339-346.

DUIT, R., 1981, Students'notion about the energy concept - before and after physics instruction. Problems concerning students'representation of physics and chemistry knowledge, Ludwigsburg.

GENZLING, J.C., et TEMME, F., 1982, Construction du concept d'énergie à l'école élémentaire, Ecole Normale d'Instituteur, Colmar.

GODSTEIN, M., HOWE, A., 1978, Application of piagetian theory to introductory chemistry instruction, Journal of Chemical Education, 55.

GOOD, R., KROMHOUT, R.A., MELLON, E.K., 1979, Piaget's work and chemical education, Journal of Chimical Education, 56, 426-430.

HULIN, M., 1982, Présentation de séquences pédagogiques sur l'énergie. Recherche sur l'Enseignement de la Notion d'Energie, INRP.

KHRAIBANI, S., 1984, Thèse de 3è cycle, Université Paris 7. Registres d'interprétation des élèves et des professeurs de collège dans le domaine de la mécanique.

LE MOIGNE, J.L., 1984, La théorie du système genéral. Theorie de la modélisation, PUF, Paris, 2ème éd.
MAC DERMOTT, L., 1984, Revue critique de la recherhe dans le domaine de la mécanique, Recherche en didactique de la physique, (Ed. du CNRS: Paris).

MARTINAND, J.L., 1984, La construction de la notion d'énergie, Compte-rendu du stage INRP de ST PRIX, 1982, ASTER $\mathrm{N}^{\circ} 20$.

MEHEUT, M., 1982, Combustion et réaction chimique dans un enseignement destiné à des élèves de sixième (11-12 ans) - Thèse de 3è cycle. Université Paris 7.

PFUNDT, H., 1981, The final link in the division process or the first building block? Pre-instruccional conceptions about the structure of substances, Chimica Didactica 7 $75-94$.

PIAGET, J., BLISS, J., Les changements d'état de la parafine d'une bougie (non publié).

PIAGET, J., INHELDER, B., 1962, Le developpment des quantites physiques, Chap. V et VI, Delachaux Niestlé, Paris, 99-130.

SERE, M.G., 1984, Apport d'un modèle particulaire à la compréhension des actions exercées par les gaz, par đes élèves de 6ème (no publicado).

SIMON, H., 1974, La science des systèmes, science de l'artificiel, Epi S.A. Editeurs, Paris.

SOLOMON, J., Pupils'background ideas about energy. Documento interno. Chelsea College, London s.d.

VIENNOT, L., 1979, La raisonnement spontané en dynamique élémentaire (Paris: Hermann).

WALLISER, B., 1977, Systèmes et modeles, introduction critique à l'analyse des systèmes, Editions du Seuit, Paris.

WATTS, D.M., 1982, Does sciences education meet the needs of today's school-leavers? A case study of the concept of energy. School teacher fellows Summer Conference. 